\title{
A Scalable Geographic Routing Protocol for Mobile Ad Hoc Network with Grid Positioning System
}

\author{
S.Aarthi \\ PG Scholar \\ Department of CSE \\ SKP Engineering College \\ Tiruvannamalai, India
}

\author{
L.Sankaran \\ Asst.Professor \\ Department of CSE \\ SKP Engineering College, \\ Tiruvannamalai, India
}

\begin{abstract}
This paper examine that the geographic routing protocols are used in the mobile ad hoc networks. In routing protocols defines that each node identify the position of one hop neighbours, and also the packets are forwarded to a neighbours that is closer to the destination. This forwarding strategy should prevent radio communications between node. However, inexpensive position devices do not provide accuracy for these algorithms and it is difficult to obtain the information on moving nodes. If the nodes should know the shape and position. It have better routing decisions so the packets that are avoided. We proposes that the grid positioning represents by grid ends that are convenient to use and also affected by a small movements of nodes. This paper presents geographic shortest path routing that are used to find out the shortest path at a particular node and also the grid partitioning of a network. The grid cells also contains the density of the nodes. When the node density increases, if the grid cells are stable. This paper also presents simulation results that evaluate the grid-based routing and compares it with position-based routing.
\end{abstract}

\section{General Terms \\ Mobile Computing}

\section{Keywords}

Geographic Routing,Grid Based Routing,Position Based Routing,Ad Hoc Networks

\section{INTRODUCTION}

In this paper, we examine location-based routing networks, nodes are developed and they move dynamically. When each node know the position and its one-hop neighbors, packets can be forwarded toward any destination in the network .Here, the geographic forwarding strategy should fails, that they prevent radio communications between nodes. As a geographic forwarding,needs a routing recovery algorithm to get around obstacles. Since all the recovery techniques require that nodes know the location of previous nodes. However, inexpensive position devices doesn't provide sufficient accuracy for these algorithms and it is difficult to obtain the information on continuously moving nodes. Our approach to position-based routing algorithm, if nodes know the shape and position, so packets are avoided. They have arbitrary complex shapes to store and process, and they change as edge nodes move. Then, the obstacles are represented on a grid map of the network with the accuracy of the grid. Our geographic routing uses the obstacle map to find the geographic shortest path that avoids intervening obstacles in [2].In addition, since the grid partitioning naturally aggregates nodes into grid elements, we can develop routing algorithms on the basis of the grid elements, not the position of individual nodes. As node density will increase, the state of grid elements becomes more stable[1][2][3]. On the basis of the grid network model, a class of grid-based algorithms for geographic routing. We present algorithms, geographic shortest path planning, path tracing and recovery. These algorithms are combined to design a full geographic shortest path routing protocol and a robotic routing protocol.

In particular, the grid-based recovery algorithm,can replace the earlier recovery techniques to improve the performance of the geographic routing protocol. The recovery algorithm avoids the recovery techniques that have being used.

Our simulations are designed to investigate the characteristics of routing algorithms. In static networks, we validate the routing algorithms by running them in a variety of scenarios. In mobile networks, the effects of node density, mobility, and beacon frequency are measured by the metrics of packet delivery rate, path length, and routing efficiency. The results should based on the grid-based routing is less affected by mobility and location errors than conventional position-based routing. The comparison with the current standard geographic routing protocol shows that our grid-based routing follows shorter paths and achieves a much higher packet delivery rate but uses smaller amount packet transmissions in mobile networks. As a example, it perform simulations on the movement of nodes. Nodes are randomly developed and each node are moves in a random manner through a position along the shortest path that avoids obstacles.

Finally,this paper also presents simulation results that evaluate the grid-based routing and compares it with position-based routing.

\section{RELATED WORKS}

\subsection{Geographic Random Forwarding}

Geographic Random Forwarding node defines that each node has its own position and velocity i.e., the node are to be delivered.While this is certainly a model for propagation, it is assumed a first step towards fundamental behaviors.For Example, Rayleigh fading. Preliminary results show that an approach for Rayleigh fading,each node that have a packet to send, it sends it using some type of broadcast address while specifying its own location and the location of the intended destination. All active nodes in the coverage area will receive this packet and will assess their own priority in trying to act as a relay, based on how close they are to the destination.

\subsection{Self Adaptive On-Demand Geographic Routing}

In On-Demand Geographic Routing assume every mobile node is its own position through some kind of location service.To improve routing performance and mobile nodes 
enable the promiscuous mode on their network interfaces.Additionally, if they consider the destination position inaccuracy and also to minimize the delivery failure.

\subsection{Geographic Routing without Location Information}

Geographic routing uses nodes locations and forwards packets in a greedy manner towards the destination.A node has no neighbor closer to the destination a variety of methods such as perimeter routing in GPSR.A method for routing around voids that is both asymptotically worst case optimal as well as average case efficient. Geographic routing is scalable, as nodes only keep state for their neighbors, and supports a fully general any-to-any communication pattern without explicit route establishment. However, geographic routing requires that nodes know their location.

\subsection{A Location Based Routing Method for Irregular Mobile Ad Hoc Networks}

Geographic routing uses location information for packet delivery in multihop wireless networks. Neighbours locally exchange location information obtained through GPS (Global Positioning System). The former measures the percentage of new neighbours a forwarding node is unaware of but that are actually within the radio range of the forwarding node on determination techniques.

\subsection{Self-Position Update For Geographic Routing in Mobile Ad Hoc Networks}

In geographic routing protocol becomes the attractive choice for the mobile ad hoc networks for that purpose many position system are involved. Position based routing protocol are mainly used in many networks due to dynamic change in the network topology and it is highly suitable for this present networks. But some of the protocol like Greedy Perimeter Stateless Routing Protocol and DSR protocol are also used.

\section{SELECTIVITY ESTIMATION 3.1 Preliminaries}

\subsubsection{Geographic Shortest Path}

Given two positions on the grid-based map of a network, shortest path algorithms that are used to finding the shortest path between the two points.In many shortest path algorithms are developed in some fields such as robotics, graphics, and computer games.It represents the shortest path algorithms that are efficient to compute of shortest path by using this grid based system.

\subsubsection{Ad hoc network}

An Ad hoc network is a Local Area Network(LAN) connection that is to be build suddenly as devices are connected. The message flows to each node in the network, the nodes are forwarded to packets with each other.It is also a wireless connection in a network.

\subsubsection{Routing}

Routing is used to selecting the most excellent path in the network.It is forwarded to network traffic between nodes. Routing is performed for many kinds of networks, Electronic data networks. In this devices that we are using in this Packet switching. Thus, constructing routing tables, which are held in the router's memory, is very important for efficient.

\subsection{Proposed Mechanism}

Several selectivity estimation methods have been proposed.Mainly it focus only on the Grid positioning
method.It will represented by the grid ends that are well suitable to use and also affected by a small movements of nodes.In this paper present geographic shortest path routing that are used to find out the shortest path at a particular node.

Routing features affect the shape of a mobile ad hoc network by the movement of nodes, mainly when the network is selforganized among people who carry a wireless communication device. For instance, lakes and rivers cause a large area in the network because peoples are moving very rare in these places. When people travelling in their vehicles, they are forming a network shaped by the road side. In cities people moving to the streets rather than in park areas. So the nodes are not uniformly developed.

We define a grid network model that have been considered by the geographic features. The grid network model have processed by the regions should have being in an empty state and gives geographic routing the potential for scalability, mobility and quality of service measures.

\subsubsection{Geographic shortest path algorithm}

Given two positions on the grid-based mapping system of a network, the shortest path algorithms finding the shortest path between the two points. Most of them shortest path algorithms have been developed in the fields of robotics, graphics, and computer games.

\subsubsection{Grid based Forwarding}

We evaluate this function that are based on this Grid positioning system.Then finally we have using this particular Algorithm for such node.This algorithm is fully based on to finding out the shortest distance.In this function that the values are assigned at each part at that current positioning system. This function that recognize each step to be varied.

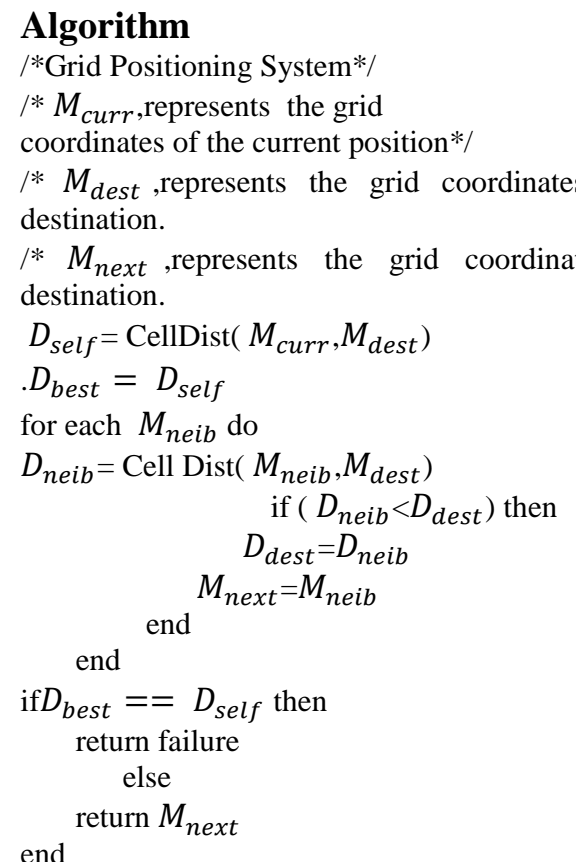

This algorithm explains that how to finding out the nearest position of that node.Since in this first step defines the current position of the neighbours and also the destination and the next hop of the particular cell. $D_{\text {self }}$ explains that in what way that the node to be reduced in that path.After that it will be get the best solution in a positioning system. 


\section{GRID POSITIONING SYSTEM ARCHITECTURE}

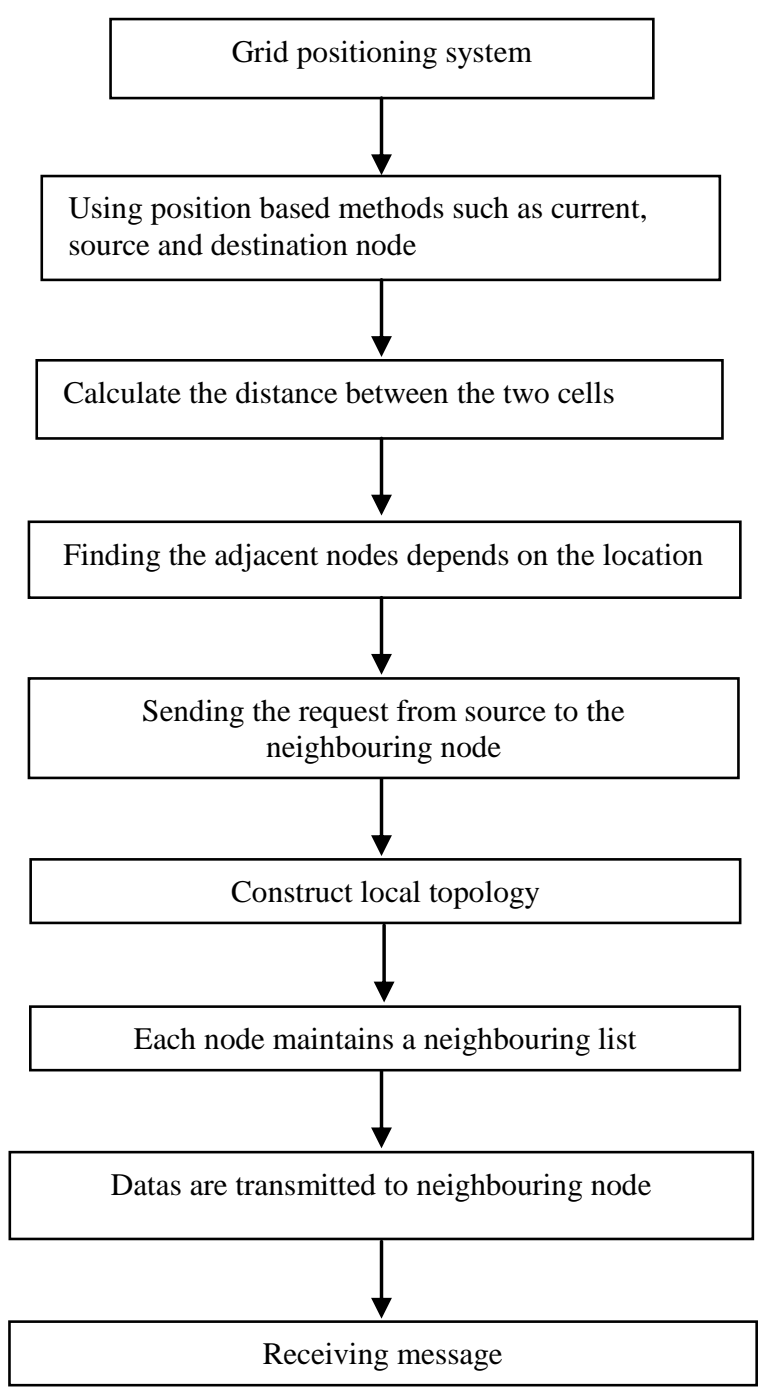

Figure 1.Grid Positioning Architecture 


\section{EXPERIMENTAL EVALUATION}

In geographic shortest path routing, the couple of packets may follow a long path as in GPSR, but the nodes are exchanging more packets, they follow a shorter path. The efficiency of our heuristic routing scheme is measured by how quickly the rules map intervening obstacles. Our simulation network is designed to evaluate this feature.

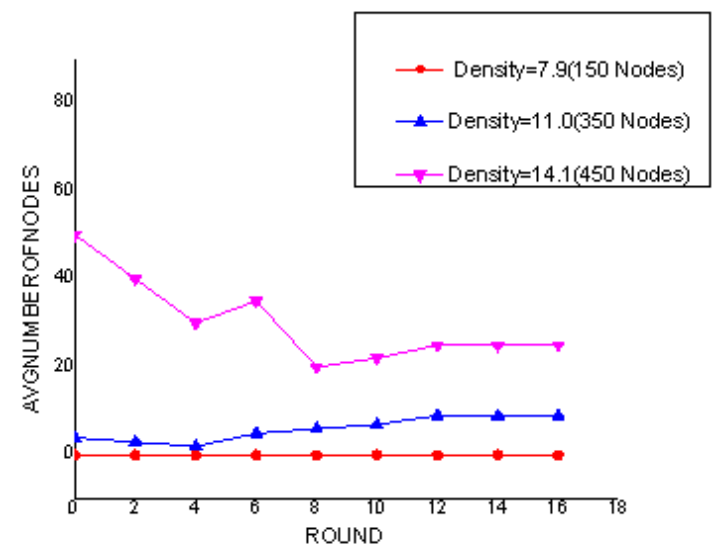

Figure 2. Packet Delivery Ratio

We randomly deploy nodes in the area of $1000 \mathrm{~m} \times 1000 \mathrm{~m}$. A source node sends a packet and the destination replies, similarly to the procedure that happens in a TCP session. Each session consists of 16 packets, which are enough to evaluate our routing scheme. We repeat this experiment for 100 different randomly generated networks.In this simulation, randomly generated large voids are obstacles.In this Lowdensity networks have been more obstacles than high-density networks. The transmission range of nodes is $100 \mathrm{~m}$.

\section{CONCLUSION}

This paper addresses solution to the estimation of Geographic routing. The obstacles represented on the grid are easy to process, and are not likely to change as frequently as the position of nodes.Based on the network model, we presented a novel geographic routing technique in which packets follow the geographic shortest path. The geographic shortest path routing technique shows how to find and use the best path in Mobile Ad hoc Networks.

\section{ACKNOWLEDGEMENTS}

The authors wish to thank Daejoong Kim,N.Nilson for the help of this work and R.Govindan for pointing this related work.

\section{REFERENCES}

[1] P. Hart, N. Nilson, and B. Raphael, 1968, "A formal basis for the heuristic determination of minimum cost paths," IEEE Transactions on Systems, Science and Cybernetics, vol. SCC-4, pp. 100107.

[2] Ding, W. and Marchionini, G. 1997 A Study on Video Browsing Strategies. Technical Report. University of Maryland at College Park.

[3] Frohlich, B. and Plate, J. 2000. The cubic mouse: a new device for three-dimensional input. In Proceedings of the SIGCHI Conference on Human Factors in Computing Systems.

[4] B. Zhou, Y.Z. Lee, M. Gerla, and F. de Rango, Oct 2006, "A scalable routing protocol for ad hoc networks with group motion,"Wireless Communications and Mobile Computing, vol. 6, no. 7, pp. 989\{1002.

[5] D.Niculescu and B.Nath, Apr. 2003 "Localized positioning in ad hoc networks," in Sensor Network Protocols and Applications, 2003.

[6] S. Datta, I. Stojmenovic, and J. Wu,2002, "Internal node and shortcut based routingwith guaranteed delivery in wireless networks," Cluster Computing, vol. 5,pp. $169\{178,2002$.

[7] C. Lochert, H. Hartenstein, J. Tian, H. Fubler, D. Hermann, and M. Mauve,2003," Arouting strategy for vehicular ad hoc networks in city environments," in Proceedings of the IEEE Intelligent Vehicles Symposium.

[8] X.-Y. Li, G. Calinescu, P.J. Wan, and Y. Wang, "Localized Delaunay triangulation with application in ad hoc wireless networks," IEEE Trans. on Paralleland Distributed Systems., vol. 14, no. 10.

[9] X. Du and D. Wu,jan 2006, “Adaptive cell-relay routing protocol for mobile ad hoc networks," IEEE Transactions on Vehicular Technology, vol. 55, no. 1, pp. 278\{285.

[10] M. Joa-Ng and I.T. Lu, Aug 1999,“A peer-to-peer zonebased two-level link state routing for mobile ad hoc networks," IEEE Journal on Selected Areas In Communication, vol. 17, no. 8,pp.1415\{142. 